\title{
Modeling Asphaltene Aggregation with a Single Compound
}

Bianca Breure ${ }^{1}$, Deepa Subramanian ${ }^{2,3}$, Jan Leys ${ }^{2,4}$, Cor J. Peters ${ }^{1}$, and Mikhail A. Anisimov ${ }^{2,3, *}$

${ }^{1}$ Chemical Engineering Department, The Petroleum Institute, Abu Dhabi, United Arab Emirates

${ }^{2}$ Institute for Physical Science and Technology, University of Maryland, College Park, United States

${ }^{3}$ Department of Chemical and Biomolecular Engineering, University of Maryland, College Park, United States

${ }^{4}$ Laboratorium voor Akoestiek en Thermische Fysica, Departement Natuurkunde en Sterrenkunde, KU Leuven, Leuven, Belgium

KEYWORDS Dynamic light scattering, model asphaltene compound, hexa-tert-butylhexaperihexabenzocoronene, precipitant-induced aggregation, diffusion-limited aggregation

\begin{abstract}
Aggregation of a model asphaltene compound hexa-tert-butylhexa-perihexabenzocoronene (HTBHBC) in toluene/heptane mixtures was monitored in real time by using Dynamic Light Scattering. The observed aggregation behavior was compared to that of real asphaltenes. It was found that heptane induces aggregation of HTBHBC and that the growth rate of the aggregates depends on the toluene/heptane ratio, closely resembling the aggregation kinetics of real asphaltenes. For the conditions studied in this work only diffusion-limited aggregation was observed. We thus confirm that a single well-defined compound can be successfully used for modeling the process of asphaltene aggregation.
\end{abstract}




\section{INTRODUCTION}

Asphaltenes are the heaviest, most aromatic and most polar fraction of crude oils. They are also the most enigmatic fraction of crude oils regards their self-assembly and stability in solutions. Asphaltenes are a focal point of "Petroleomics" [1], which is the prediction of behavior of petroleum and petroleum fractions based on molecular structure of the components, whose complete list is named "petroleome”, a "fingerprint” of petroleum.

Asphaltenes are usually defined as a solubility class: they are the petroleum fraction that is insoluble in alkanes, such as pentane or heptane, but soluble in aromatic solvents such as benzene or toluene [2]. Asphaltenes cause many problems during oil production, transport and processing as they have the tendency to precipitate and deposit upon changes in pressure, temperature, or composition [3]. Over the past decades, many research efforts have focused on gaining a better understanding of the phase behavior and aggregation behavior of this petroleum fraction. One of the techniques, that are used to study the kinetics of asphaltene aggregation, is Photon Correlation Spectroscopy, also known as Dynamic Light Scattering (DLS). Anisimov et al. [4] used DLS to study the aggregation and flocculation of asphaltenes extracted from Arabian and Venezuelan crude oils in solutions of varying toluene/heptane ratio. They found that the kinetics of asphaltene aggregation-flocculation can be divided into three stages: (i) a nucleation stage in which asphaltene clusters of a critical size are formed, (ii) an aggregation stage in which these clusters grow into basic aggregates and (iii) a flocculation stage, in which flocks with a fractal structure are formed from the basic aggregates. Yudin et al. [5] continued this research with asphaltenes from Karazhanbas crude oil, but with a special focus on the aggregation stage. They demonstrated that the aggregation kinetics of asphaltene particles can be either reactionlimited or diffusion-limited, depending on the initial asphaltene concentration in toluene. For 
intermediate concentrations they also observed a crossover between these two regimes. Burya et al. [6] studied the growth of asphaltene aggregates in three different types of crude oils, originating from different sources, upon addition of the precipitant heptane with a DLS technique that was specially adapted for opaque, almost optically nontransparent liquids. They observed that asphaltene behavior strongly depends on the nature of the oil, in particular, on the amount of paraffins, and on the amount of heptane added. These results have been reviewed by Yudin and Anisimov [7]. Espinat et al. [8] studied the effects of temperature and pressure on asphaltene aggregation with light, X-ray and neutron scattering. By using light scattering, they studied the effect of temperature on the aggregation of Safaniya asphaltenes dissolved in toluene. They observed a considerable increase in the hydrodynamic radius of the asphaltene aggregates when the temperature was decreased from $273 \mathrm{~K}$, to $263 \mathrm{~K}$ to $253 \mathrm{~K}$. At $253 \mathrm{~K}$ the asphaltene aggregates started to sediment. DLS has also been used to study the effect of stabilizing compounds on asphaltene aggregation and flocculation. Hashmi and Firoozabadi studied the effect of amphiphilic [9] and polymeric dispersants [10] on the aggregation behavior of asphaltenes upon addition of heptane, whereas Heaps et al. [11] investigated the influence of naphthenic acids. In all cases it was found that the stabilizers delay the onset of aggregation. These works illustrate that DLS is a well-suited technique to study aggregation of asphaltenes.

Since asphaltenes are defined as a solubility class, it is a fraction that covers a range of different components. This range is highly dependent on the origin of the crude oil and also on the method and the precipitant that are used for asphaltene extraction. Hence, asphaltenes are a complex, polydisperse and ill-defined group of different components. This has mainly contributed to the fact that our current knowledge of asphaltene behavior is still limited. The aim of the present work is to get a better understanding of real asphaltenes by studying a well-defined 
model system containing a single asphaltene-like compound. By studying different model compounds with well-defined molecular structures one will be able to achieve the main goal of Petroleomics, making a link between the molecular structure and observed behavior. This will lead to a better understanding of the more complex real asphaltenes found in crude oils.

In this work we present results obtained with a model asphaltene compound hexa-tertbutylhexa-perihexabenzocoronene (HTBHBC). The molecular structure of HTBHBC is shown in Figure 1. HTBHBC is a graphene-like molecule with features common to real asphaltenes, namely its large aromatic core and aliphatic side chains. In addition, HTBHBC and its derivatives are known to self-assemble in solution, likely due to interactions between the aromatic cores (so-called $\pi-\pi$ stacking) $[12,13]$. We have performed experiments to study the effect of the addition of the precipitant, heptane, to solutions of HTBHBC in toluene. Heptane is a strong precipitant for real asphaltenes and one can expect a similar effect in the solutions of HTBHBC.

\section{EXPERIMENTAL SECTION}

\section{Sample Preparation}

Experiments were performed with a single compound HTBHBC (serving as an asphaltene model) and with an asphaltene sample extracted from North Sea crude oil. The asphaltenes were extracted according to a modified IP-143 procedure [14]: $40 \mathrm{ml}$ of pentane was added to $1 \mathrm{~g}$ of crude oil and mixed before the mixture was left to settle for at least $14 \mathrm{~h}$. The sample was then centrifuged and the supernatant liquid was decanted. The asphaltene residue was washed several times with pentane until the solvent stayed colorless. HTBHBC was 
synthesized according to the procedure described by Rathore and Burns [15]. Its purity was checked with HPLC and ${ }^{1} \mathrm{H}$ NMR and was estimated to be greater than $90 \%$.

Samples were prepared by adding heptane to an initial solution of either HTBHBC or asphaltenes in toluene. Toluene (Sigma Aldrich 99.9\% purity) was used as the solvent and heptane (Sigma-Aldrich, 99\% purity) as the precipitant. The initial solutions contained about 0.5 wt\% HTBHBC in toluene and about $0.03 \mathrm{wt} \%$ asphaltenes in toluene. Lower concentrations of real asphaltenes in toluene were used to reduce the opacity of the system. Before the start of each experiment, the initial solution in toluene and the heptane were each filtered with $200 \mathrm{~nm}$ nylon Millipore filters to remove dust and insoluble impurities. Optical cells were used for the DLS experiments, which were first washed with isopropyl alcohol and then dried with high purity dust-free nitrogen gas (Airgas). At the start of an experiment the optical cell was filled with a known amount of HTBHBC-toluene solution or asphaltene-toluene solution. A known amount of heptane was added to this solution. The optical cell was shortly manually shaken and then placed in the DLS set-up; the measurements were started immediately. This way, samples with three different ratios of toluene to heptane were prepared for both HTBHBC and asphaltenes.

\section{Light-Scattering Technique and Procedure}

A Photocor Instruments light-scattering setup was used for performing all the experiments. It consists of a He-Ne laser, a photomultiplier tube, and an automatic goniometer. A detailed description of the set-up can be obtained in ref. [16]. The optical cell was placed in an immersion liquid (silicone oil with a refractive index that closely matches that of the optical cell) to reduce spurious scattering and improve temperature control. All measurements were carried out at room temperature $\left(25.7{ }^{\circ} \mathrm{C} \pm 0.2{ }^{\circ} \mathrm{C}\right)$ and ambient pressure. Time-dependent intensity 
autocorrelation functions were obtained with a Photocor "multitau" correlator, with a logarithmic time distribution. In previous light scattering studies of asphaltene aggregation a linear correlator was used [7]. Measurements were performed at four different angles $\left(45^{\circ}, 60^{\circ}, 90^{\circ}\right.$ and $\left.120^{\circ}\right)$. The accumulation time was chosen as a compromise between an "as-short-as-possible" time to ensure no significant change in the aggregate size occurs during the measurement interval and a sufficient accumulation time needed to get a good signal-to-noise ratio. The accumulation time at each angle was 1 minute while the total duration of each experimental run varied between 2.5 and 5 hours.

For single exponential decay relaxation modes, the intensity auto-correlation function $g_{2}(t)$ obtained in the homodyning mode is given by [17]:

$$
g_{2}(t)-1=C \exp \left[-2\left(\frac{t_{\mathrm{lag}}}{\tau}\right)\right] \text {, }
$$

where $C$ is the amplitude, $t_{\text {lag }}$ is the "lag" (or "delay") time of the photon correlations and $\tau$ is the characteristic diffusion time of aggregates, assumed to be Brownian particles. For Brownian particles, the diffusion time is related to the diffusion coefficient, $D$, as follows:

$$
D=\frac{1}{\tau q^{2}},
$$

where $q$ is the difference in wave number between incident and scattered light, $q=(4 \pi n / \lambda) \sin (\theta / 2), n$ is the refractive index of the solvent, $\lambda$ is the wavelength of the incident light in vacuum and $\theta$ is the scattering angle. For monodisperse, spherical Brownian particles the hydrodynamic radius $R$ can be calculated by using the Stokes-Einstein relation: 


$$
R=\frac{k_{\mathrm{B}} T}{6 \pi \eta D}
$$

where $k_{\mathrm{B}}$ is Boltzmann's constant, $T$ is the temperature and $\eta$ is the shear viscosity of the medium.

\section{RESULTS AND DISCUSSION}

Colloidal particles can grow according to two mechanisms [18,19]: (i) diffusion-limited aggregation (DLA), where the aggregation rate is determined by the time it takes for particles to collide via Brownian motion and (ii) reaction-limited aggregation (RLA), where the growth rate is limited by the probability of forming a bond upon collision of two particles. When particles grow according to a DLA process, their hydrodynamic radius is a function of time $t$ reads [19]:

$$
R=A_{R} t^{1 / d_{\mathrm{f}}}+R_{0}
$$

where $A_{R}$ is an effective aggregation rate constant, $d_{\mathrm{f}}$ the fractal dimension and $R_{0}$ is the initial size of the particle. The kinetics of RLA is described by the following formula [19]:

$$
R=R_{0}^{\prime} \exp \left[t / \tau_{R L A}\right]^{1 / d_{\mathrm{f}}},
$$

where $\tau_{\mathrm{RLA}}$ is the characteristic time of the exponential reaction-limited aggregation and $R_{0}$ is the initial size of the particle. The above equations can also be written in terms of diffusion time $\tau$, assuming the viscosity of the solution does not significantly change during the aggregation process. For DLA, this leads to: 


$$
\tau=A_{\tau} t^{1 / d_{\mathrm{f}}}+\tau_{0}
$$

where $A_{\tau}$ is an effective aggregation rate constant and $\tau_{0}$ is the characteristic diffusion time of the initial particle. For RLA, the equation for diffusion time $\tau$ can be written as:

$$
\tau=\tau_{0} \exp \left[t / \tau_{\mathrm{RLA}}\right]^{1 / d_{\mathrm{f}}}
$$

The fractal dimension in the above equations is a measure of how compactly an object fills its space. It is a measure of complexity comparing how details in a pattern change with the scale [20,21]. The aggregation of colloidal particles by DLA or RLA mechanism shows universal behavior leading to formation of fractal structures [19]. Aggregation of colloids by DLA mechanism leads to structures with a fractal dimension of $1.75 \pm 0.05$, while aggregation by RLA mechanism lead to structures with a fractal dimension of $2.05 \pm 0.05$ [22-24]. Aggregation of asphaltenes also follows this universal behavior leading to formation of fractal structures [7].

The correlation functions for all of the angles studied were fitted to exponential functions of the following form

$$
g_{2}(t)-1=C \exp \left[-2\left(\frac{t_{\text {lag }}}{\tau}\right)^{\beta}\right]
$$

where $\beta$ is a "stretch/anti-stretch" exponent. The values of $\beta$ smaller than one could be an indication of the presence of polydisperse particles resulting in stretched exponential decays of the auto-correlation function. In all our fits, the value of $\beta$ is obtained to be $1 \pm 0.4$. Due to 
marginal variations in fit quality, obtained for different values of $\beta$, we interpret our correlation functions as being roughly single exponentials.

Figure 2 presents the diffusion time $\tau$ as a function of aggregation time $t$, obtained at four different scattering angles, for the model compound HTBHBC in a heptane/toluene solution (0.74 mole fraction heptane). The diffusion times are calculated by fitting Eq. (8) to the measured intensity auto-correlation functions. The solid lines are fits to Eq. (6). The fractal dimension was fixed at 1.75 , which is the expected fractal dimension for diffusion-limited aggregation [19]. The negative curvature (clearly not an exponential growth) and good match between experimental data and the power law fits are an indication that the kinetics of aggregation is indeed controlled by diffusion. Dispersion interactions between aromatic rings may be a sufficient mechanism for aggregation in HTBHBC. It should be noted that due to the experimental procedure, there is a small time period of approximately two minutes between sample preparation and the start of the measurements. As a result, the initial nucleation stage could not be detected and thus $\tau_{0}$ in our experiments corresponds to the diffusion time of aggregates that have grown to a critical nucleus of size $R_{0}$.

Figure 3 shows the hydrodynamic radius $R$ as a function of aggregation time $t$, in HTBHBC solutions at scattering angle $60^{\circ}$. The hydrodynamic radius was obtained from $\tau$, in accordance with Eq. (8) and with use of Eqs. (2) and (3). The refractive index and viscosity of the medium was obtained by a linear interpolation between the refractive indices and viscosites of pure toluene and heptane (based on mole fractions). The solid lines are fits to Eq. (4). The fractal dimension in Eq. (4) was fixed at 1.75. Data are shown for three different concentrations of heptane/toluene mixtures, namely, 0.70, 0.74, and 0.80 mole fractions of heptane. From this 
figure, it is seen that as the amount of heptane increases, the rate of aggregation also increases. Thus heptane is a strong precipitant for enhancing the aggregation of HTBHBC.

Figure 4 shows the hydrodynamic radius for asphaltene solutions at scattering of angle $60^{\circ}$. The solid lines are fits to Eq. (4) with a fractal dimension of 1.75. Data are shown for three different concentrations of heptane/toluene mixtures, namely, $0.58,0.66$, and 0.74 mole fraction of heptane. As the concentration of heptane increases, the rate of aggregation of asphaltenes increases. The similarity between Figures 3 and 4 indicate that asphaltene aggregation behavior in toluene/heptane solutions under DLA conditions can be reasonably modeled by model compound HTBHBC.

In Table 1 , we summarize the average values for $A_{R}$ and $R_{0}$ for HTBHBC and asphaltenes, obtained by fitting $T$ to Eq. (6) and then by using Eqs. (2) and (3). The fitting parameter $A_{R}$ is an effective aggregation-rate constant and from Table 1 we observe that this number is greater in asphaltenes than it is in HTBHBC, indicating that aggregation in asphaltenes is faster than in HTBHBC. The parameter $R_{0}$ in our experiments is the size of the initial aggregate formed after the nucleation stage. From Table 1, we observe that the initial size in asphaltenes is greater than in HTBHBC, supporting the argument that the aggregation rate is faster in asphaltenes than in HTBHBC. It is also seen that as the amount of heptane increases, the values of $A$ and $R_{0}$ also increase, thus confirming that addition of more precipitant, namely heptane, enhances aggregation in asphaltenes and HTBHBC. 
Table 1: The fitting parameters for HTBHBC and Asphaltenes

\begin{tabular}{|c|c|c|c|c|c|}
\hline \multicolumn{3}{|c|}{ HTBHBC } & \multicolumn{3}{c|}{ Asphaltenes } \\
\hline Heptane & $A_{R}$ & $R_{0}$ & Heptane & $A_{R}$ & $\left(\times 10^{-8}\right)$ \\
$($ mole & $\left(\times 10^{-8}\right)$ & $\left(\times 10^{-6}\right)$ & $($ mole & $\left(0^{-6}\right)$ \\
fraction) & $\left(\mathrm{m} \cdot \mathrm{s}^{-1 / 1.75}\right)$ & $(\mathrm{m})$ & fraction $)$ & $\left(\mathrm{m} \cdot \mathrm{s}^{-1 / 1.75}\right)$ & $(\mathrm{m})$ \\
& & & & & 0.090 \\
\hline 0.70 & 0.065 & 0.104 & 0.58 & 0.240 & 0.237 \\
\hline 0.74 & 0.082 & 0.111 & 0.66 & 0.246 & 0.274 \\
\hline 0.80 & 0.102 & 0.133 & 0.74 & 0.362 & \\
\hline
\end{tabular}

\section{CONCLUSIONS}

In this work we compare the aggregation of the model compound HTBHBC and real asphaltenes dissolved in toluene upon addition of the precipitant heptane. It is observed that the model compound HTBHBC shows aggregation behavior similar to real asphaltenes. In both systems heptane induces the formation and growth of aggregates. It is observed that the diffusion time of the auto-correlation functions and the hydrodynamic radius of the particles increases according to a power law during the experimental run, which is an indication that aggregation is a diffusion-limited process. We have described the experimental data for both real asphaltenes and the model compound with diffusion-limited aggregation kinetics, leading to aggregates with a fractal dimension of 1.75, which is typical for classical colloids. Future experiments will focus on studying more model asphaltene-like compounds with a variety of side chains including polar groups. It will be interesting to investigate whether a crossover from diffusion-limited aggregation to reaction-limited aggregation, which is observed for real asphaltenes if their 
concentration increases [5,7], as well as in some classical colloids [18,19], can also be found for the asphaltene-like model components. The effect of polydispersity of the aggregates on the aggregation kinetics is another important problem for further studies.

\section{AUTHOR INFORMATION}

\section{Corresponding Author}

* Email: anisimov@umd.edu. Tel.: + 1-301-405-8049. Fax: +1-301-314-9404.

\section{ACKNOWLEDGMENT}

This research is supported by a grant from the Research Initiation Funding Program 2012 of The Petroleum Institute, Abu Dhabi, United Arab Emirates and by the Division of Chemistry of the National Science Foundation (grant no. CHE-1012052), USA. J.L. acknowledges the Research Foundation-Flanders (FWO) for a travel grant. We would like to thank J. van Spronsen for helping us with the synthesis of the model asphaltene compound HTBHBC, S. Soroush for providing the asphaltene sample, and S. Kim and D. Weglein for help with light-scattering measurements and data analysis. We also acknowledge interactions with I. K. Yudin of Photocor Instruments on dynamic light-scattering techniques. 


\section{REFERENCES}

(1) Asphaltenes, Heavy Oils and Petroleomics. Mullins, O.C.; Sheu, E.Y.; Hammami, A.; Marshall, A.G.; Eds. Springer: New York, 2007.

(2) The Chemistry and Technology of Petroleum. Speight, J.G. Marcel Dekker, New York, 1999.

(3) Leontaritis, K.J. Asphaltene deposition: a comprehensive description of problem manifestations and modeling approaches, In: Proceedings of the SPE Production Operations Symposium, Oklahoma City, USA, March 1989, Vol. 13/14, 599-609.

(4) Anisimov, M.A.; Yudin, I.K.; Nikitin, V.; Nikolaenko, G.; Chernoutsan, A. Asphaltene aggregation in hydrocarbon solutions studied by photon correlation spectroscopy. J. Phys. Chem. 1995, 99, 9576-9580.

(5) Yudin, I.K.; Nikolaenko, G.L.; Gorodetskii, E.E.; Markhashov, E.L.; Agayan, V.A.; Anisimov, M.A.; Sengers, J.V. Crossover kinetics of asphaltene aggregation in hydrocarbon solutions, Physica A 1998, 251, 235-244.

(6) Burya, Y.G.; Yudin, I.K.; Dechabo, V.A.; Anisimov, M.A. Colloidal properties of crude oils studied by dynamic light-scattering, Int. J. Thermophys. 2001, 22, 1397-1410.

(7) Yudin, I.K.; Anisimov, M.A. Dynamic light scattering monitoring of asphaltene aggregation in crude oils and hydrocarbon solutions. Chapter 17: Asphaltenes, Heavy Oils and Petroleomics. Mullins, O.C.; Sheu, E.Y.; Hammami, A.; Marshall, A.G.; Eds. Springer: New York, 2007;

(8) Espinat D.; Fenistein, D.; Barré, L.; Frot, B.; Briolant, Y. Effects of temperature and pressure on asphaltenes agglomeration in toluene. A light, x-ray and neutron scattering investigation. Energy Fuels 2004, 18, 1243-1249.

(9) Hashmi, S.M.; Firoozabadi, A. Effect of dispersant on asphaltene suspension dynamics: aggregation and sedimentation. J. Phys. Chem. B 2010, 114, 15780-15788. 
(10) Hashmi, S.M.; Firoozabadi, A. Tuning size and electrostatics in non-polar colloidal asphaltene suspensions by polymeric adsorption. Soft Matter 2011, 7, 8384-8391.

(11) Heaps, D.T.; Madasu, P.K.; Magers, D.H.; Buchanan, J.P. Investigation of the precipitation behavior of asphaltenes in the presence of naphtenic acids using light scattering and molecular modeling techniques, Energy Fuels 2012, 26, 1862-1869.

(12) Hill, J.P.; Jin, W.; Kosaka, A.; Fukushima, T.; Ichihara, H.; Shimomura, T.; Ito, K.; Hashizume, T.; Ishii, N.; Aida, T. Self-assembled hexa-peri-hexabenzocoronene graphitic nanotube, Science 2004, 304, 1481-1483.

(13) Wu, J.; Fechtenkötter, A.; Gauss, J.; Watson, M.D.; Kastler, M.; Fechtenkötter, C.; Wagner, M.; Müllen, K. Controlled self-assembly of hexa-peri-hexabenzocoronenes in solution, J. Am. Chem. Soc. 2004, 126, 11311.

(14) IP 143/90 Asphaltene (heptane insolubles) in Petroleum Products, In: Standards for Petroleum and its Products, Institute of Petroleum, London, John Wiley \& Sons, 1985, pp 143.1143.7

(15) Rathore, R.; Burns, C.L. A practical one-pot synthesis of soluble hexa-perihexabenzocoronene and isolation of its cation-radical salt, J. Org. Chem. 2003, 68, 4071-4074.

(16) Subramanian, D.; Ivanov, D. A.; Yudin, I. K.; Anisimov, M. A.; Sengers, J. V. Mesoscale inhomogeneities in aqueous solutions of 3-methylpyridine and tertiary butyl alcohol. J. Chem. Eng. Data 2011, 56, 1238-1248.

(17) Dynamic Light Scattering: With Applications to Chemistry, Biology, and Physics. Berne, B.J.; Pecora, R.; Wiley: New York, 1976.

(18) Weitz, D.A.; Huang, J.S.; Lin, M.Y.; Sung, J.; Limits of the fractal dimension for irreversible kinetic aggregation of gold colloids, Phys. Rev. Lett. 1985, 54 (13), 1416-1419. 
(19) Lin, M.Y.; Lindsay, H.M.; Weitz, D.A.; Ball, R.C.; Klein, R.; Meakin, P. Universality in colloid aggregation, Nature 1989, 339, 360-339.

(20) Fractal Geometry: Mathematical Foundations and Applications. Falconer, K.; John Wiley \& Sons: England, 2003.

(21) The Fractal Geometry of Nature. Mandelbrot, B. B.; W.H. Freeman \& Company: New York, 1983.

(22) Witten, T. A.; Sander, L. M. Diffusion-limited aggregation, a kinetic critical phenomenon. Phys. Rev. Lett. 1981, 47, 1400-1403.

(23) Meakin, P. Formation of fractal clusters and networks by irreversible diffusion-limited aggregation. Phys. Rev. Lett. 1983, 51, 1119-1122.

(24) Meakin, P.; Family, F. Structure and kinetics of reaction-limited aggregation. Phys. Rev. A 1988, 38, 2110-2123. 


\section{Figures}

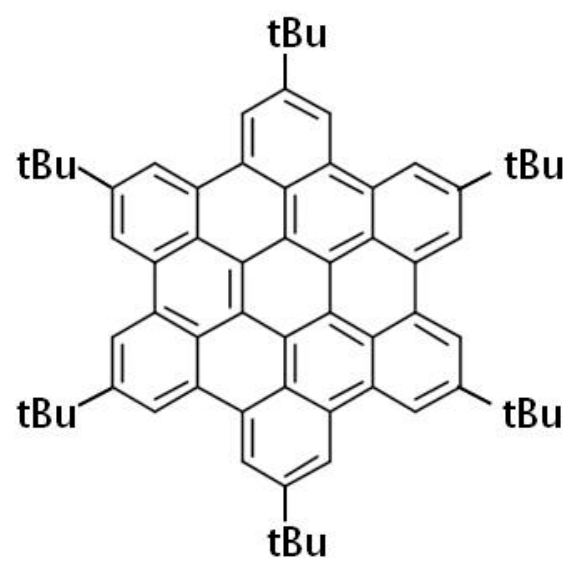

Figure 1. Molecular structure of hexa-tert-butylhexa-perihexabenzocoronene (HTBHBC) $\left(\mathrm{C}_{66} \mathrm{H}_{66}, \mathrm{M}_{\mathrm{w}}=859.2 \mathrm{~g} / \mathrm{mol}\right)$ 


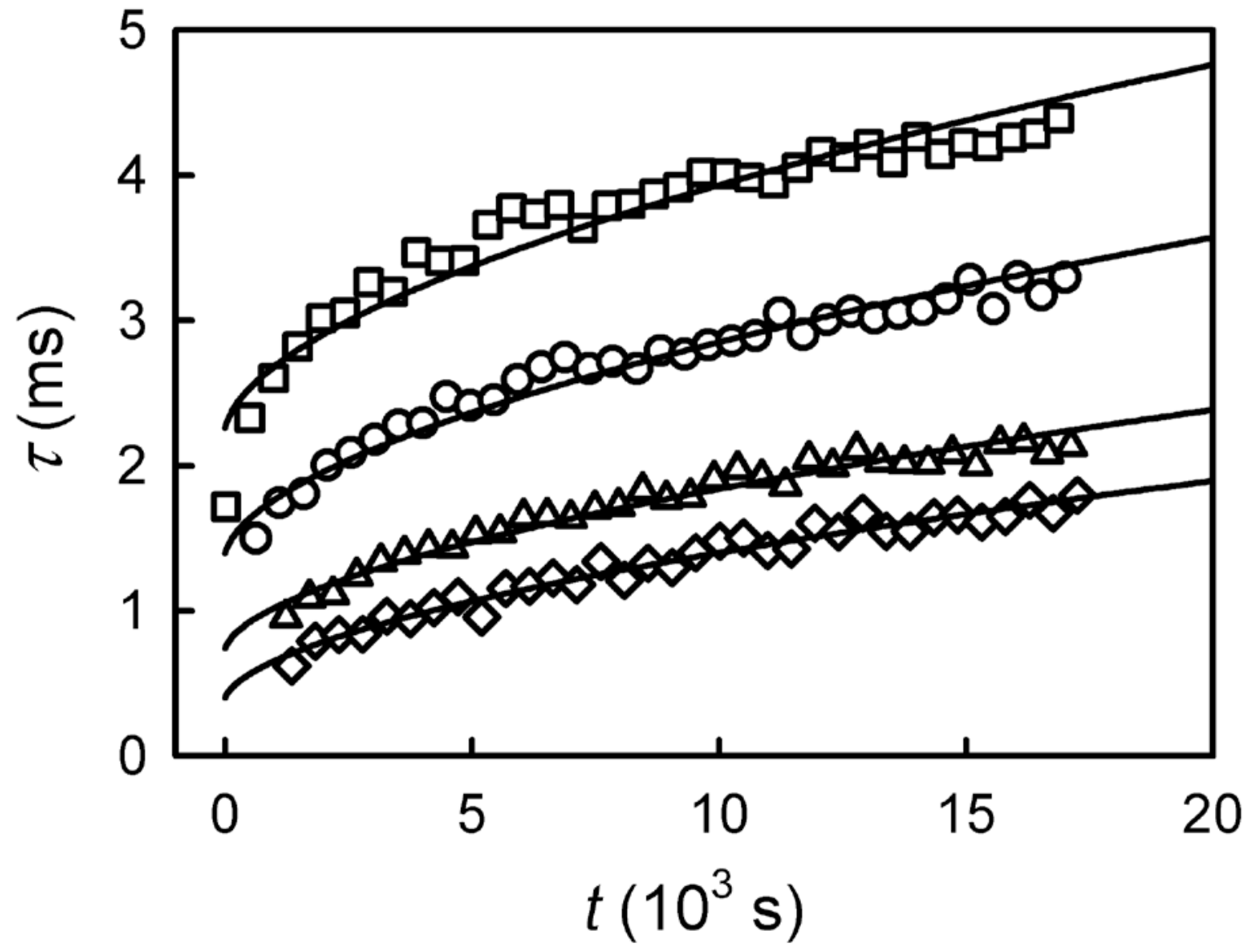

Figure 2: Diffusion time as a function of aggregation time for HTBHBC in heptane / toluene solution (0.74 mole fraction of heptane) at various scattering angles. The solid lines are fits to Eq. (6). $T=25.7^{\circ} \mathrm{C}$. Squares $=45^{\circ}$; Circles $=60^{\circ}$; Triangles $=90^{\circ}$; Diamonds $=120^{\circ}$. 


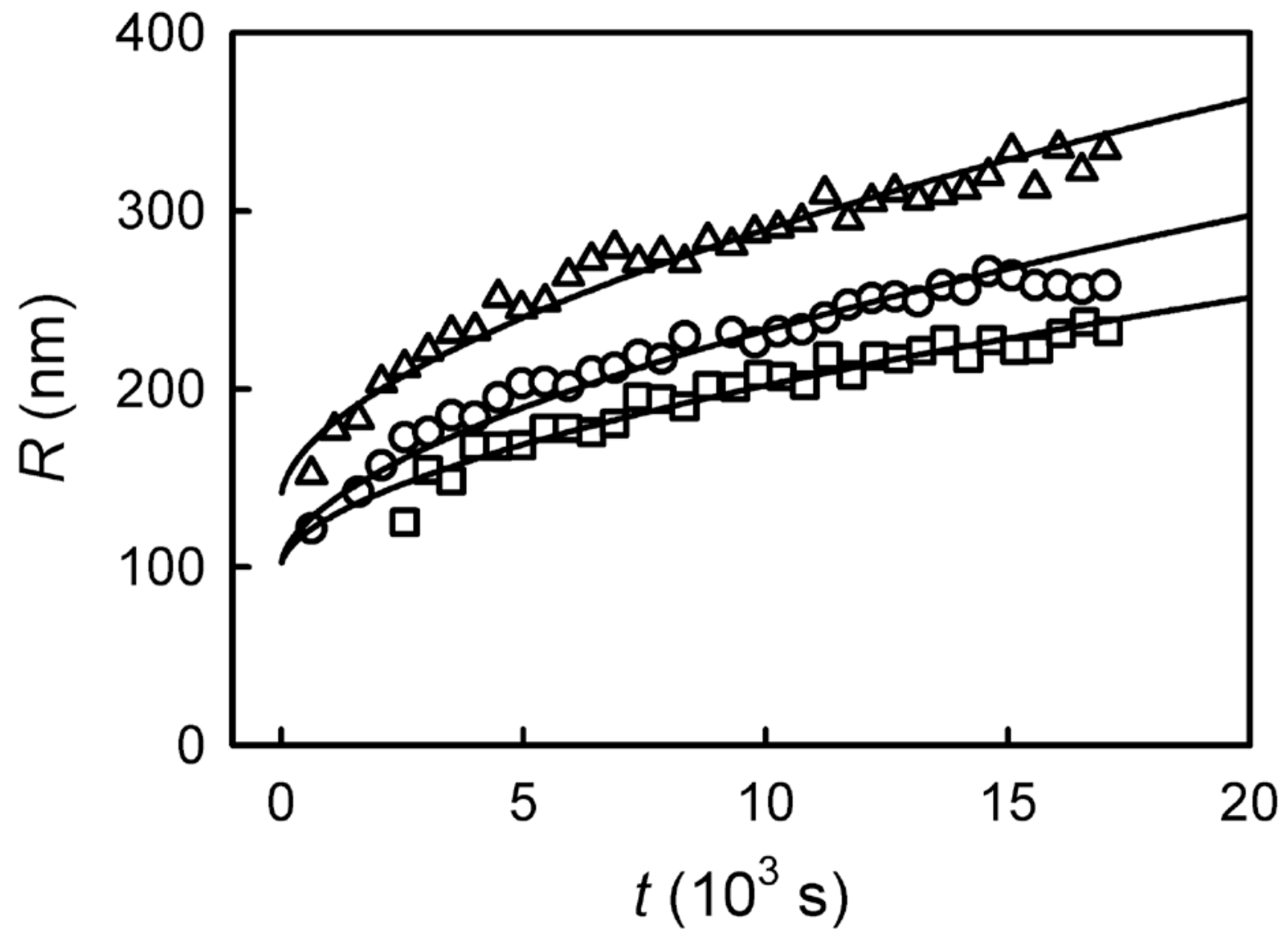

Figure 3: Hydrodynamic radius as a function of aggregation time for HTBHBC at scattering angle $60^{\circ}$. Data are for three different heptane/toluene solvent compositions. The solid lines are fits to Eq. (6) with use of Eqs. (2) and (3). $T=25.7^{\circ} \mathrm{C}$.

Mole fraction heptane: Squares - 0.70; Circles - 0.74; Triangles - 0.80 . 


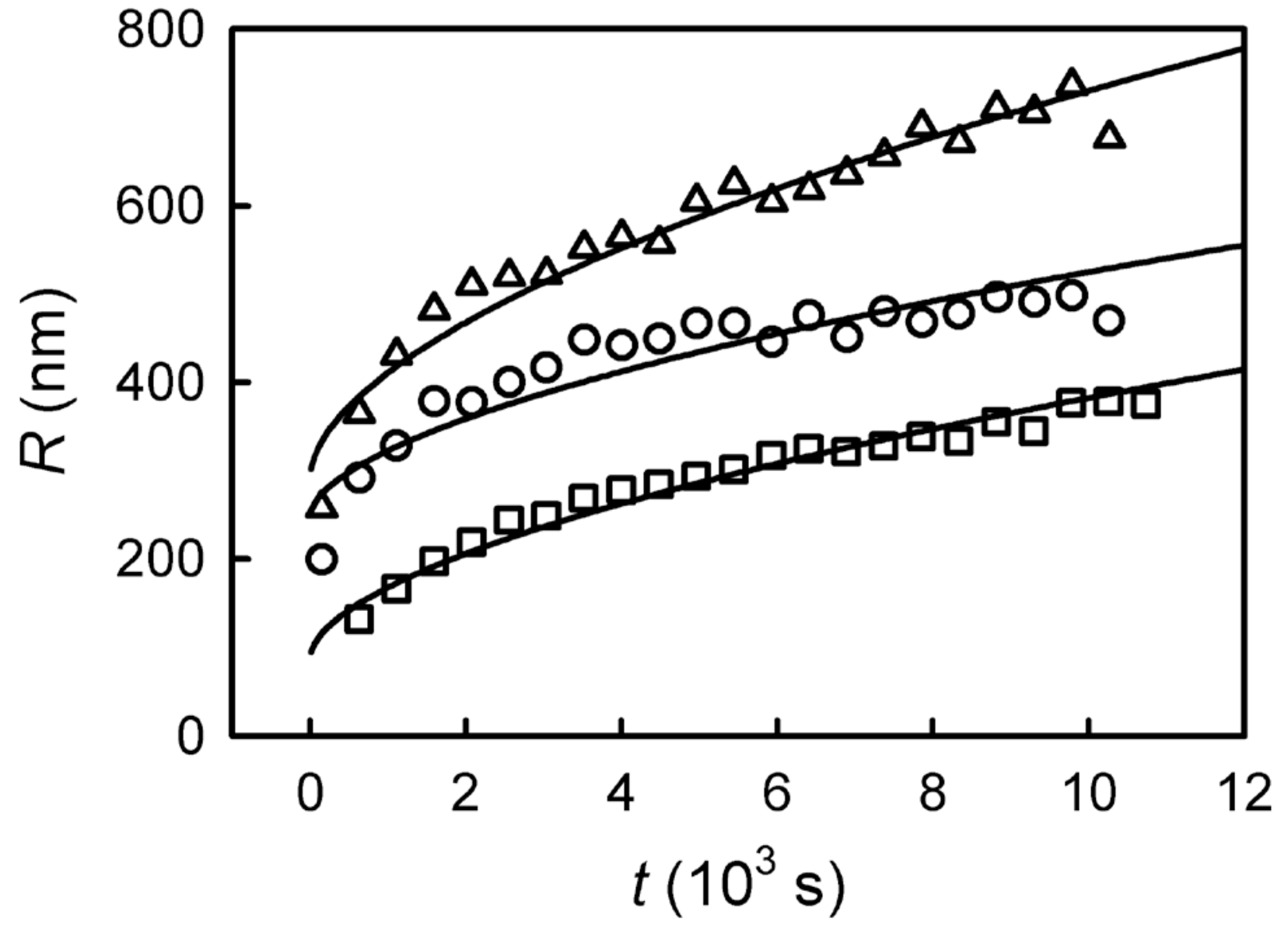

Figure 4: Hydrodynamic radius as a function of aggregation time for asphaltenes at scattering angle $60^{\circ}$. Data are for three different heptane/toluene solvent compositions. The solid lines are fits to Eq. (6) with use of Eqs. (2) and (3). $T=25.7^{\circ} \mathrm{C}$.

Mole fraction of heptane: Squares - 0.58; Circles - 0.66; Triangles - 0.74. 